\title{
ON SUBALGEBRA LATTICES OF UNIVERSAL ALGEBRAS
}

\section{A. A. ISKANDER ${ }^{1}$}

Abstract. If $\boldsymbol{A}$ is a universal algebra, $S(A)$ is the lattice of all subalgebras of $A$. If $B \subseteq A \times A, B^{*}$ is $\{(x, y):(y, x) \in B\}$.

THEOREM. Let $L_{1}, L_{2}, L_{3}$ be algebraic lattices such that $\left|L_{1}\right|$, $\left|L_{2}\right|>1$. Let $\alpha_{i}$ be an involutive automorphism of $L_{i}, i=1,2$. Then there are two universal algebras $A_{1}, A_{2}$ of the same similarity type, having the properties:

(a) there are lattice isomorphisms $\beta_{i}$ of $L_{i}$ onto $S\left(A_{i} \times A_{i}\right), i=1,2$, and $\beta_{3}$ of $L_{3}$ onto $S\left(A_{1} \times A_{2}\right)$;

(b) $\left(l \alpha_{i}\right) \beta_{i}=\left(l \beta_{i}\right)^{*}, l \in L_{i}, i=1,2$.

The lattices of all subalgebras of universal algebras were characterized by G. Birkhoff and O. Frink in [1] as algebraic lattices (see also [2], [3] for terminology). In [4] the author proved that every algebraic lattice is isomorphic to the subalgebra lattice of the square of some universal algebra. A simpler proof of this result, not using the axiom of choice, was given by $\mathrm{G}$. Grätzer and W. A. Lampe in [3].

If $\boldsymbol{A}$ is a universal algebra denote by $\mathscr{S}(\boldsymbol{A})$ the subalgebra lattice of $\boldsymbol{A}$. If $B \in \mathscr{S}(A \times A)$ then $B^{*}=\{(x, y):(y, x) \in B\}$. The aim of this note is to show the following generalization of the main result in [4].

TheOREM. Let $L_{1}, L_{2}, L_{3}$ be algebraic lattices such that $\left|L_{1}\right|,\left|L_{2}\right|>1$. Let $\alpha_{i}$ be an involutive automorphism of $L_{i}, i=1,2$. Then there are two universal algebras $A_{1}, A_{2}$ of the same similarity type, having the properties:

(a) there are lattice isomorphisms $\beta_{i}$ of $\boldsymbol{L}_{i}$ onto $\mathscr{S}\left(\boldsymbol{A}_{i} \times \boldsymbol{A}_{i}\right), i=1,2$, and $\beta_{3}$ of $L_{3}$ onto $\mathscr{S}\left(A_{1} \times A_{2}\right)$;

(b) $\left(l \alpha_{i}\right) \beta_{i}=\left(l \beta_{i}\right)^{*}, l \in L_{i}, i=1,2$.

The case where one of $\left|L_{1}\right|,\left|L_{2}\right|$ is 1 is equivalent to the characterization of the connection between $\mathscr{S}(\boldsymbol{A})$ and $\mathscr{S}(\boldsymbol{A} \times \boldsymbol{A})$. This problem for partial universal algebras was solved by the author in [5]. For the case of full universal algebras the question remains open.

We will give here the construction of the algebras $A_{1}, A_{2}$. The details of the proof are similar to those of [3] and therefore will be omitted.

Received by the editors May 4, 1971.

AMS 1970 subject classifications. Primary 08A25.

Key words and phrases. Algebraic lattice, compact elements, universal algebra, subalgebra, involutive automorphism, partial operations, free extensions.

1 Research supported by National Science Foundation grant GP-29129. 
If $A=\langle A ; F\rangle$ is a partial algebra, by $\mathscr{S}(A)$ will be denoted the lattice of all (closed) subalgebras of $A$ and by $\mathscr{C}(A)$ the family of all finitely generated subalgebras of $A$. The set of all subsets of a set $X$ will be denoted by $P(X)$.

Lemma 1. Let $L_{1}, L_{2}, L_{3}, \alpha_{1}, \alpha_{2}$ be as in the theorem. Then there are partial algebras $\left\langle B_{1} ; F\right\rangle$ and $\left\langle B_{2} ; F\right\rangle$ with the properties:

(i) there are isomorphisms $\beta_{i}$ of $\boldsymbol{L}_{i}$ onto $\mathscr{S}\left(\boldsymbol{B}_{i} \times \boldsymbol{B}_{i}\right), i=1,2$, and $\beta_{3}$ of $L_{3}$ onto $\mathscr{S}\left(B_{1} \times B_{2}\right)$;

(ii) $\left(l \alpha_{i}\right) \beta_{i}=\left(l \beta_{i}\right)^{*}, l \in L_{i}, i=1,2$;

(iii) all partial operations in $F$ are injective on $B_{1}, B_{2}$, i.e. $f\left(a_{1}, \cdots, a_{n}\right)=$ $f\left(b_{1}, \cdots, b_{n}\right)$ implies $a_{1}=b_{1}, \cdots, a_{n}=b_{n}$;

(iv) there are mappings $\gamma_{i}$ of $\mathscr{C}\left(\boldsymbol{B}_{i} \times \boldsymbol{B}_{i}\right)$ into $B_{i} \times B_{i}, i=1,2$, and $\gamma_{3}$ of $\mathscr{C}\left(\boldsymbol{B}_{1} \times \boldsymbol{B}_{2}\right)$ into $B_{1} \times B_{2}$ such that every finitely generated subalgebra is generated by one element: namely its image under the appropriate $\gamma_{1}, \gamma_{2}, \gamma_{3}$.

Let $C_{j}$ denote the set of all compact elements in $\boldsymbol{L}_{j}, j=1,2,3$ (consider the $L_{j}$ mutually disjoint). Let $B$ be an infinite set strictly containing $C_{1} \cup C_{2} \cup C_{3}$. Choose $B_{1}, B_{2}$, two disjoint copies of $B$. If $b \in B$, denote by $b_{i}$ the element of $B_{i}$ corresponding to $b$ (under a fixed bijection from $B$ onto $\left.B_{i}\right), i=1,2$. Since $\left|L_{i}\right|>1$ we have $\left|C_{i}\right|>1$. Choose $c_{0}^{i} \in C_{i}, c_{0}^{i} \neq 0_{i}$ (the zero element of $\left.L_{i}\right)$ and $c_{0}^{i} \alpha_{i}=c_{0}^{i}, i=1,2$. Fix $b_{0} \in B \backslash\left(C_{1} \cup C_{2} \cup C_{3}\right)$.

Define mappings $\delta_{1}, \delta_{2}, \delta_{3}$, from $C_{1}, C_{2}, C_{3}$ into $P\left(B_{1} \times B_{1}\right), P\left(B_{2} \times B_{2}\right)$ and $P\left(B_{1} \times B_{2}\right)$ respectively:

For $i=1,2$,

$$
\begin{aligned}
0_{i} \delta_{i} & =\left\{\left(b_{i}, b_{i}\right): b \in B\right\}, \\
c \delta_{i} & =\left\{\left(c_{i},\left(c \alpha_{i}\right)_{i}\right)\right\} \quad \text { if } c \in C_{i}, c \alpha_{i} \neq c, \\
c \delta_{i} & =\left\{\left(c_{i}, b_{0 i}\right),\left(b_{0 i}, c_{i}\right)\right\} \quad \text { if } c \in C_{i}, c \alpha_{i}=c, c \neq 0_{i}, c \neq c_{0}^{i}, \\
c_{0}^{i} \delta_{i} & \left.=B_{i} \times B_{i}\right) \bigcup\left\{c \delta_{i}: c \in C_{i}, c \neq c_{0}^{i}\right\} ; \\
c \delta_{3} & =\left\{\left(c_{1}, b_{02}\right)\right\}, c \in C_{3}, c \neq 0_{3}, \\
0_{3} \delta_{3} & =B_{1} \times B_{2} \mid \bigcup\left\{c \delta_{3}: c \in C_{3}, c \neq 0_{3}\right\} .
\end{aligned}
$$

The following properties of $\delta_{1}, \delta_{2}, \delta_{3}$ are obvious:

1. $c \delta_{j} \neq \varnothing$ for all $c \in C_{j}, j=1,2,3$.

2. If $c, c^{\prime} \in C_{j}, c \neq c^{\prime}$ then $c \delta_{j} \cap c^{\prime} \delta_{j}=\varnothing, j=1,2,3$.

3. $\bigcup\left\{c \delta_{i}: c \in C_{i}\right\}=B_{i} \times B_{i}, i=1,2$.

4. $\bigcup\left\{c \delta_{3}: c \in C_{3}\right\}=B_{1} \times B_{2}$.

Define a family $F=F_{0} \cup F_{1} \cup F_{2} \cup F_{3}$ of partial operations on $B_{1}, B_{2}$ as follows:

To every $b \in B$, associate $f_{b} \in F_{0}-$ a nullary operation, the result of which, on $B_{i}$, is $b_{i}, i=1,2$.

To every $c, c^{\prime}, c^{\prime \prime} \in C_{i}$ such that each of $c, c^{\prime}, c^{\prime \prime}$ is distinct from $0_{i}$ and 
$c \leqq c^{\prime} \vee c^{\prime \prime}$ and to every $(a, b) \in c \delta_{i},\left(a^{\prime}, b^{\prime}\right) \in c^{\prime} \delta_{i}$ and $\left(a^{\prime \prime}, b^{\prime \prime}\right) \in c^{\prime \prime} \delta_{i}$, associate a binary partial operation $f \in F_{i}$ such that

and

$$
f\left(a^{\prime}, a^{\prime \prime}\right)=a, \quad f\left(b^{\prime}, b^{\prime \prime}\right)=b
$$

$$
D\left(f, B_{i}\right)=\left\{\left(a^{\prime}, a^{\prime \prime}\right),\left(b^{\prime}, b^{\prime \prime}\right)\right\}, \quad D\left(f, B_{k}\right)=\varnothing \quad \text { if } k \neq i, i=1,2 .
$$

To every $c, c^{\prime}, c^{\prime \prime} \in C_{3}$ and to every $a, a^{\prime}, a^{\prime \prime} \in B_{1}$ and $b, b^{\prime}, b^{\prime \prime} \in B_{2}$ such that $(a, b) \in c \delta_{3}, \quad\left(a^{\prime}, b^{\prime}\right) \in c^{\prime} \delta_{3}$ and $\left(a^{\prime \prime}, b^{\prime \prime}\right) \in c^{\prime \prime} \delta_{3}$, associate a binary partial operation $g \in F_{3}$ such that

and

$$
g\left(a^{\prime}, a^{\prime \prime}\right)=a, \quad g\left(b^{\prime}, b^{\prime \prime}\right)=b
$$

$$
D\left(g, B_{1}\right)=\left\{\left(a^{\prime}, a^{\prime \prime}\right)\right\}, \quad D\left(g, B_{2}\right)=\left\{\left(b^{\prime}, b^{\prime \prime}\right)\right\} .
$$

It can be verified that the operations of $F$ are injective on $B_{1}, B_{2}$. The binary operations in $F_{3}$ are defined only on $B_{1} \times B_{2}$, on the diagonals of $B_{1} \times B_{1}$ and $B_{2} \times B_{2}$ and nowhere else.

The isomorphisms $\beta_{1}, \beta_{2}, \beta_{3}$ are defined by

$$
l \beta_{j}=\bigcup\left\{c \delta_{j}: c \in C_{j}, c \leqq l\right\}, \quad l \in L_{j}, j=1,2,3 .
$$

It can be shown that $\left\langle B_{1} ; F\right\rangle,\left\langle B_{2} ; F\right\rangle$ satisfy conditions (i) and (ii) of Lemma 1.

Since a subalgebra of a partial algebra $\boldsymbol{A}$ is finitely generated iff it is compact in $\mathscr{S}(\boldsymbol{A})$, define $\gamma_{1}, \gamma_{2}, \gamma_{3}$ by:

If $i=1,2$,

$$
\begin{aligned}
\left(0_{i} \beta_{i}\right) \gamma_{i} & =\left(0_{i i}, 0_{i i}\right), \\
\left(c \beta_{i}\right) \gamma_{i} & =\left(c_{i},\left(c \alpha_{i}\right)_{i}\right) \quad \text { if } c \in C_{i}, c \alpha_{i} \neq c, \\
& =\left(c_{i}, b_{0 i}\right) \quad \text { if } c \in C_{i}, c \alpha_{i}=c, c \neq 0_{i}, \\
\left(0_{3} \beta_{3}\right) \gamma_{3} & =\left(0_{31}, 0_{32}\right), \\
\left(c \beta_{3}\right) \gamma_{3} & =\left(c_{1}, b_{02}\right) \quad \text { if } c \in C_{3}, c \neq 0_{3} .
\end{aligned}
$$

It can be shown that $\gamma_{1}, \gamma_{2}, \gamma_{3}$ satisfy (iv) of Lemma 1 .

If $\boldsymbol{B}$ is a partial algebra, denote by $\boldsymbol{B}^{1}$ the free algebra generated by $\boldsymbol{B}$; we have:

Lemma 2. Let $\left\langle B_{i} ; F\right\rangle, i=1,2$, be partial algebras satisfying all the conditions of Lemma 1. Then

(i) the operations of $F$ are injective on $B_{1}^{1}$ and $B_{2}^{1}$;

(ii) if $D \in \mathscr{S}\left(\boldsymbol{B}_{i} \times \boldsymbol{B}_{i}\right)$ and $\bar{D}$ is the subalgebra of $\boldsymbol{B}_{i}^{1} \times \boldsymbol{B}_{i}^{1}$ generated by $D$ then $\bar{D} \cap\left(B_{i} \times B_{i}\right)=D, i=1,2$;

(iii) if $D \in \mathscr{S}\left(\boldsymbol{B}_{1} \times \boldsymbol{B}_{2}\right)$ and $\bar{D}$ is the subalgebra of $\boldsymbol{B}_{1}^{\mathbf{1}} \times \boldsymbol{B}_{2}^{1}$ generated by $D$ then $\bar{D} \cap\left(B_{1} \times B_{2}\right)=D$; 
(iv) $D \rightarrow$ the subalgebra of $\boldsymbol{B}_{i}^{1} \times \boldsymbol{B}_{i}^{1}$ generated by $D$ is a lattice monomorphism of $\mathscr{S}\left(\boldsymbol{B}_{i} \times \boldsymbol{B}_{i}\right)$ into $\mathscr{S}\left(\boldsymbol{B}_{i}^{1} \times \boldsymbol{B}_{i}^{1}\right), i=1,2$;

(v) $D \rightarrow$ the subalgebra of $B_{1}^{1} \times B_{2}^{1}$ generated by $D$ is a lattice monomorphism of $\mathscr{S}\left(\boldsymbol{B}_{1} \times \boldsymbol{B}_{2}\right)$ into $\mathscr{S}\left(\boldsymbol{B}_{1}^{1} \times \boldsymbol{B}_{2}^{1}\right)$;

(vi) if a belongs to the subalgebra of $B_{i}^{1} \times B_{i}^{1}$ generated by $B_{i} \times B_{i}$ there exists an $\bar{a} \in B_{i} \times B_{i}$ such that for $D \in \mathscr{S}\left(B_{i} \times B_{i}\right)$, a belongs to the subalgebra of $\boldsymbol{B}_{i}^{1} \times \boldsymbol{B}_{i}^{1}$ generated by $D$ iff $\bar{a} \in D, i=1,2$;

(vii) if a belongs to the subalgebra of $B_{1}^{1} \times B_{2}^{1}$ generated by $B_{1} \times B_{2}$, there exists an $\bar{a} \in B_{1} \times B_{2}$ such that for $D \in \mathscr{S}\left(B_{1} \times B_{2}\right)$, a belongs to the subalgebra of $B_{1}^{1} \times B_{2}^{1}$ generated by $D$ iff $\bar{a} \in D$.

LEMMA 3. Let $\left\langle B_{1} ; F\right\rangle,\left\langle B_{2} ; F\right\rangle$ be partial algebras satisfying all the conditions of Lemma 1. Then one can define two families of partial operations $F^{\prime}, F^{\prime \prime}$ on $B_{1}^{1}, B_{2}^{1}$ such that the partial algebras $B_{i}^{\prime}=\left\langle B_{i}^{1} ; F^{1}\right\rangle, i=1,2$, $F^{1}=F \cup F^{\prime} \cup F^{\prime \prime}$, enjoy all the properties in Lemma 1, and properties (i), (ii) of Lemma 2, where $B_{i}^{1}$ should be replaced by $B_{i}^{\prime}, i=1,2$; moreover

(i) $D \rightarrow$ the subalgebra of $\boldsymbol{B}_{i}^{\prime} \times \boldsymbol{B}_{i}^{\prime}$ generated by $D$ is a lattice isomorphism of $\mathscr{S}\left(\boldsymbol{B}_{i} \times \boldsymbol{B}_{i}\right)$ onto $\mathscr{S}\left(\boldsymbol{B}_{i}^{\prime} \times \boldsymbol{B}_{i}^{\prime}\right), i=1,2$;

(ii) $D \rightarrow$ the subalgebra of $\boldsymbol{B}_{1}^{\prime} \times \boldsymbol{B}_{2}^{\prime}$ generated by $D$ is a lattice isomorphism of $\mathscr{S}\left(\boldsymbol{B}_{1} \times \boldsymbol{B}_{2}\right)$ onto $\mathscr{S}\left(\boldsymbol{B}_{1}^{\prime} \times \boldsymbol{B}_{2}^{\prime}\right)$.

For any $a, \bar{a}$ as in Lemma 2 , if $a=\left(a^{\prime}, a^{\prime \prime}\right), \bar{a}=\left(\bar{a}^{\prime}, \bar{a}^{\prime \prime}\right)$ introduce $g \in F^{\prime}$ :

$$
D\left(g, B_{1}^{1}\right) \cup D\left(g, B_{2}^{1}\right)=\left\{a^{\prime}, a^{\prime \prime}\right\} \quad \text { and } \quad g\left(a^{\prime}\right)=\bar{a}^{\prime}, \quad g\left(a^{\prime \prime}\right)=\bar{a}^{\prime \prime} .
$$

For any $d=\left(d^{\prime}, d^{\prime \prime}\right)$ not belonging to the subalgebra of $\boldsymbol{B}_{i}^{1} \times \boldsymbol{B}_{i}^{1}$ generated by $B_{i} \times B_{i}, d \in B_{i}^{1} \times B_{i}^{1}$, define $h, k \in F^{\prime \prime}$ by

$$
\begin{aligned}
D\left(h, B_{j}^{\mathbf{1}}\right) & =D\left(k, B_{j}^{\mathbf{1}}\right)=\varnothing \quad \text { if } j \neq i, \\
D\left(h, B_{i}^{\mathbf{1}}\right) & =\left\{d^{\prime}, d^{\prime \prime}\right\} \quad \text { and } \\
h\left(d^{\prime}\right) & =c_{0 i}^{i}, \quad h\left(d^{\prime \prime}\right)=b_{0 i}, \\
D\left(k, B_{i}^{\mathbf{1}}\right) & =\left\{c_{0 i}^{i}, b_{0 i}\right\} \quad \text { and } \\
k\left(c_{0 i}^{i}\right) & =d^{\prime}, \quad k\left(b_{0 i}\right)=d^{\prime \prime}, \quad i=1,2 .
\end{aligned}
$$

For any $d=\left(d^{\prime}, d^{\prime \prime}\right) \in B_{1}^{1} \times B_{2}^{1}$, not belonging to the subalgebra of $B_{1}^{1} \times B_{2}^{1}$ generated by $B_{1} \times B_{2}$ introduce $h, k \in F^{\prime \prime}$ by

$$
\begin{aligned}
D\left(h, B_{1}^{1}\right) & =\left\{d^{\prime}\right\}, & D\left(h, B_{2}^{1}\right) & =\left\{d^{\prime \prime}\right\}, \\
h\left(d^{\prime}\right) & =0_{11}, & h\left(d^{\prime \prime}\right) & =0_{22}, \\
D\left(k, B_{1}^{1}\right) & =\left\{0_{11}\right\}, & D\left(k, B_{2}^{1}\right) & =\left\{0_{22}\right\}, \\
k\left(0_{11}\right) & =d^{\prime}, & k\left(0_{22}\right) & =d^{\prime \prime} .
\end{aligned}
$$

It can be verified that $\left\langle B_{i}^{1} ; F^{1}\right\rangle, i=1,2$, enjoy all the properties of Lemma 3. 
Define the sequence of partial algebras $\left\langle A_{i n} ; G_{n}\right\rangle, i=1,2, n=0,1,2, \cdots$, by

$$
\begin{gathered}
A_{i 0}=B_{i} \quad \text { and } \quad G_{0}=F \quad(\text { of Lemma 1), } \\
A_{i(n+1)}=\left(A_{i n}\right)^{1}, \quad G_{n+1}=\left(G_{n}\right)^{1} .
\end{gathered}
$$

Put $A_{i}=\bigcup\left\{A_{i n}: n=0,1,2, \cdots\right\}, i=1,2, G=\bigcup\left\{G_{n}: n=0,1,2, \cdots\right\} .\left\langle A_{i} ; G\right\rangle$, $i=1,2$, are full universal algebras satisfying the conditions (a), (b) of the theorem.

I would like to express my thanks to the referee for reading the original manuscript and for his generous comments.

\section{REFERENCES}

1. G. Birkhoff and O. Frink, Representations of lattices by sets, Trans. Amer. Math. Soc. 64 (1948), 299-316. MR 10, 279.

2. G. Grätzer, Universal algebra, Van Nostrand, Princeton, N.J., 1968. MR 40 \#1320.

3. G. Grätzer and W. A. Lampe, On subalgebra lattices of universal algebras, J. Algebra 7 (1967), 263-270. MR 36 \#1377.

4. A. A. Iskander, Correspondence lattices of universal algebras, Izv. Akad. Nauk SSSR Ser. Mat. 29 (1965), 1357-1372. (Russian) MR 33 \#7289.

5. - Partial universal algebras with preassigned lattices of subalgebras and correspondences, Mat. Sb. 70 (112) (1966), 438-456; English transl., Amer. Math. Soc. Transl. (2) 94 (1970), 137-158. MR 33 \#5541.

Department of Mathematics, Vanderbilt University, Nashville, Tennessee 37203

Current address: Department of Mathematics, University of Southwestern Louisiana, Lafayette, Louisiana 70501 